Presented at the 18th International IUPAP Conference on Few-Body Problems in Physics, Santos, Sao Paulo, Brazil, August 21-26, 2006

\title{
Exploring the Spin Structure of the Proton with Two-Body Partonic Scattering at RHIC
}

\author{
J. Sowinski for the STAR Collaboration \\ Indiana University Cyclotron Facility and Department of Phyiscs \\ 2401 Milo B. Sampson Ln. \\ Bloomington, Indiana 47408 \\ sowinski@indiana.edu
}

The STAR collaboration at the Relativistic Heavy Ion Collider is using polarized proton beams at $\sqrt{s}=200 \mathrm{GeV}$ to study the spin structure of the proton. The first results for the double spin helicity dependence of inclusive jet production are presented along with projections for additional data taken in 2005 and 2006. When fully analyzed these data sets should place strong constraints on the possible contribution of gluonic spin to the proton spin as expressed by $\Delta \mathrm{G}$. Future studies using 2-jet or photon-jet coincidences to map out the gluon spin distribution vs. the gluon's momentum fraction of the proton are discussed.

\section{Introduction}

It would seem that the question of the contribution of 3 valence quarks to the spin of the nucleon should be a relatively straight forward 3-body problem. And in a constituent quark model, the magnetic moments of the baryon octet are explained [1] by 3 parameters representing the magnetic moments of the up, down and strange quarks, thus fully accounting for the spin of the nucleon. Ever since the first polarized deep inelastic scattering (DIS) measurements on the proton directly measured the polarization of the quarks, we have been confronted with a puzzle in that the quarks, as described in the infinite momentum frame, seem to account for at most $1 / 3$ of the proton's spin[2]. Of course in perturbative QCD (pQCD) the picture of the proton is a many-body problem, with a large number of virtual gluons and sea quarks popping in and out of existence. Current efforts are directed at trying to learn about contributions to the proton's spin from gluon spin and orbital angular momentum of the quarks and gluons. DIS measurements are sensitive to the gluons only through scaling violations, requiring global fits to the full kinematic range of the measurements and, up to now [3], have not provided strong constraints on the gluon contribution, $\Delta \mathrm{G}$.

At the Relativistic Heavy Ion Collider (RHIC) we are investigating the spin of gluons in the proton via two-body hard scattering of quarks and gluons [4, analogous to what we nuclear physicists call quasi-free scattering. The source of the gluons and quarks are $\sqrt{s}=200 \mathrm{GeV}$ polarized proton collisions in RHIC. Processes that are sensitive to the spin of the gluons include simple scattering, $q+g \rightarrow q+g$ and $g+g \rightarrow g+g$. The two 
outgoing partons fragment to produce colorless particles, mesons and baryons, correlated in space in a cone and known as a jet. Of course these processes sensitive to the gluon polarization are mixed with $q q$ and $q \bar{q}$ scattering, partially diluting the signal of interest. Sensitivity to the partonic spin is gained through the longitudinal double spin asymmetry also known as the spin correlation parameter,

$$
A_{L L}=\frac{\sigma^{++}-\sigma^{+-}}{\sigma^{++}+\sigma^{+-}}
$$

where $\sigma^{++}$refers to the cross section when both beams have the same helicity and $\sigma^{+-}$ the case where they have opposite helicity. This quantity is large for many partonic scattering processes giving good sensitivity to the spin of the partons involved in the scattering. Underlying the formalism are the familiar assumptions that the scattering is "free", that final and initial state interactions are small or calculable, and that the 2-body cross section, the spin dependent momentum distributions and the fragmentation are factorizable, i.e. do not depend on each other. The cross sections for inclusive jets are relatively large and have been the channel first studied at RHIC as the luminosity has developed in the initial years of polarized proton operation. The performance of RHIC in 2006 gives us optimism to proceed in future years with studies of smaller cross section channels such as quark-gluon Compton scattering $q+g \rightarrow q+\gamma$, which has some advantages that will be discussed below.

\section{STAR Measurements}

The inclusive jet measurements I will present were taken with the STAR detector at RHIC. STAR sits at one of six interaction regions in the $3.8 \mathrm{~km}$ circumference double ring collider. The other interaction regions are populated by Phenix which is the other large detector and heavily involved in the spin program, Phobos, Brahms, pp2pp and polarimetry. Spin runs so far have primarily consisted of two $100 \mathrm{GeV}$ polarized proton beams colliding at the center of STAR and PHENIX plus other regions as required. Test runs up to beam energies of $250 \mathrm{GeV}$ have been made and this will eventually be an important running energy. Polarization and luminosity have steadily increased[5] year to year, with the polarization reaching $60 \%$ compared to a design value of $70 \%$, and luminosity within a factor of 3 of the design goal for $\sqrt{s}=200 \mathrm{GeV}$ running in 2006 .

STAR [6], which stands for the Solenoidal Tracker at RHIC, is a large volume solenoidal magnet $(B=0.5 \mathrm{~T})$ with axis along the beam line with most of its volume filled with a time projection chamber which provides tracking and momentum analysis of charged particles. The other detector subsystems of interest for the results presented here are scintillators near the beam line at each end of the magnet, known as beam-beam counters, used for triggering, local polarimetry and luminosity monitoring, and Pb-plastic scintillator electromagnetic (EM) calorimeters covering a range approximately $15^{\circ}$ to $140^{\circ}$ with respect to the beam line. These detectors have essentially complete coverage in $\phi$ giving STAR the large solid angle coverage necessary to detect the multiple charged particles and decay photons making up a jet which can span a cone of radius $60^{\circ}$, and moreover provides the coincident solid angle to detect back to back dijets or photon-jet coincidences.

Jets are readily identified by eye in event displays of large solid angle detectors such as STAR as groups of tracked charged particles and photons from decayed mesons in the EM 

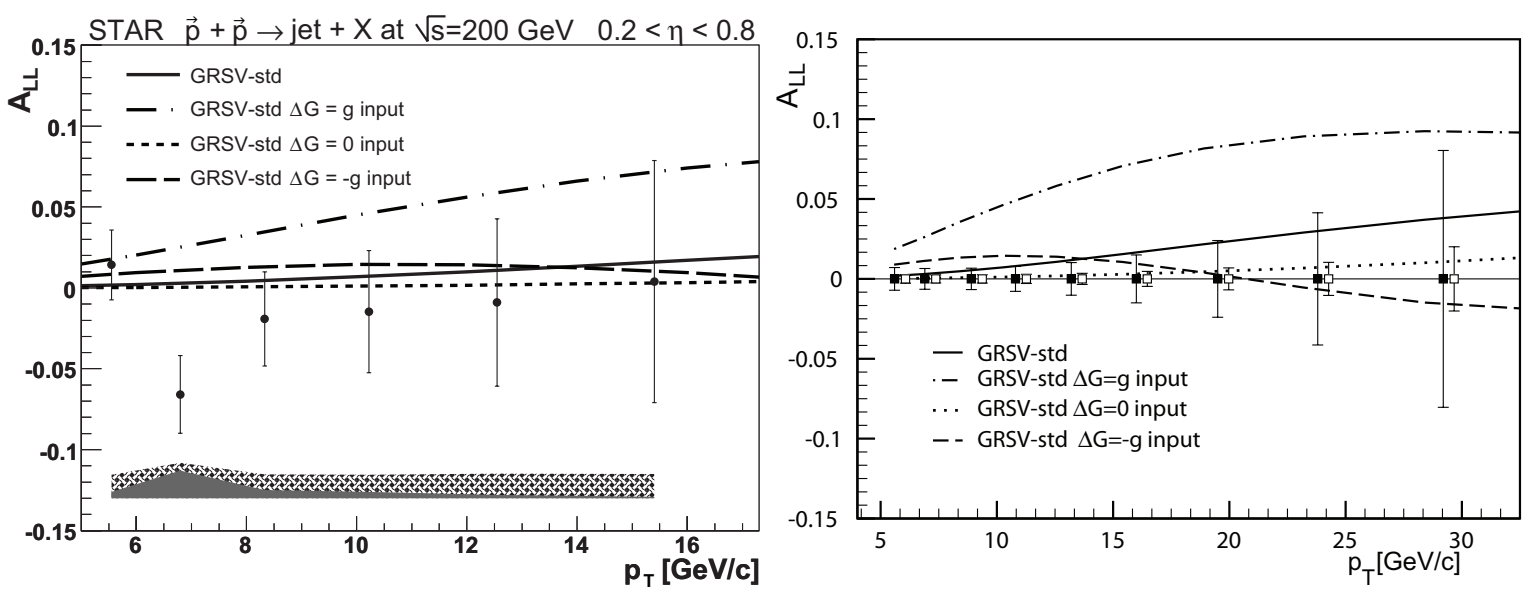

Figure 1. $A_{L L}$ for combined 2003 and 2004 inclusive jet data (left) along with the projected statistical precision for data taken in 2005 and 2006 (right).

calorimeters grouped in a cone. Particle - particle correlations also show the jet nature and their disappearance in heavy ion collisions has been important in establishing the new form of matter discovered at RHIC[7]. STAR uses the EM component of the jets in the calorimeters to trigger on candidate jet events. To do a quantitative comparison between jet data and pQCD calculations a common jet definition, an algorithm for finding jets, must be agreed upon. We are currently using a midpoint-cone algorithm developed [8] in work at the Tevatron. Individual particles above a certain transverse momentum are used as seeds to establish a cone and the energy in that cone is collected. There is a method to merge and split the cones, finally arriving at a stable definition of jets for the event. In the 2003-2004 data set, with only about 1/3 of the EM calorimetry participating, we typically find at most one jet in an event. In 2006 with full EM calorimetry, events with jets have two jets in the acceptance over $50 \%$ of the time. Events with 3 or more jets are also seen and are expected when hard gluons are radiated during the partonic scattering or fragmentation.

In the left portion of Fig. 1 are shown our results for the combined 2003 and 2004 longitudinal spin correlation $A_{L L}$ for inclusive jets recently submitted for publication [9]. The data are plotted vs. the magnitude of the momentum transverse to the beam, $\mathrm{p}_{T}$, which is a measure of the scale the collision probes much as momentum transfer is used in electron scattering. The cross section results extracted from this data compare favorably with next to leading order pQCD calculations over many orders of magnitude, particularly in slope. With the cross section giving encouragement that pQCD applies to the jets we are seeing, we compare to next-to-leading-order pQCD calculations [10] with different values of the gluonic contribution to the proton's spin, $\Delta \mathrm{G}$. The different calculations shown, span the range of allowed values, from fully polarized along the protons helicity, $\Delta \mathrm{G}=\mathrm{g}$, to fully anti-aligned, $\Delta \mathrm{G}=$-g. The GRSV std. calculation (solid line) uses a polarized gluon distribution typical of best fits to DIS data. This first $\mathrm{A}_{L L}$ inclusive jet data set rules out only the largest values of $\Delta \mathrm{G}$, and in particular $\Delta \mathrm{G}=\mathrm{g}$ at the $98 \%$ c.l.

In addition to the data presented here we have taken data sets for inclusive jets in 2005 
with improved beam luminosity and polarization and again in 2006 with further improved beam properties and the full calorimeter solid angle active and in the trigger. The size of the error bars expected for 2005 and 2006 are presented in the right hand portion of Fig. 2. The 2005 data will be available by the fall of 2006 .

One difficulty with the inclusive jet results is that each data point averages over a large range of the gluon distribution in Bjorken $x$, the fraction of the proton's momentum carried by the gluon in the infinite momentum frame. For example it would be difficult to determine whether a small $\Delta \mathrm{G}$ comes from a value uniformly small in $x$ or a distribution that changes signs. By detecting 2 jets or a gamma and jet in coincidence we have the capability in STAR to map out the $x$ dependence. The measured scattering angles are well correlated with the partonic scattering angles and ratio of the 2 partonic momentum fractions, $x_{a} / x_{b}$. The gamma-jet coincidences are particularly attractive, despite their smaller cross section, in that they strongly select for $q g$ interactions. In addition, the gamma $\mathrm{p}_{T}$ better preserves the partonic level $p_{T}$ scale than the jets, where the energy scale is smeared in fragmentation and detection. Exploiting these kinematics allows one to extract the individual $x_{q}$ and $x_{g}$ in the regime where one of them is significantly larger than the other and thus most likely to be identified with the quark. The goal in future running at $200 \mathrm{GeV}$ is to measure $\Delta \mathrm{G}(\mathrm{x})$ for $0.03 \leq \mathrm{x} \leq 0.3$. Additional running at $500 \mathrm{GeV}$ should allow us to extend to lower $x$ values and provide important cross checks where the data sets overlap.

\section{Summary}

RHIC and STAR have made a lot of progress over the past 6 years and we are starting to contribute in a significant way to an accounting of the proton's spin. Data already taken should place significant constraints on the magnitude of the gluon's contribution to the spin of the proton. There are other measurements we are performing, with transversely polarized beams for example, I have not had time to discuss which are making important contributions 11 to other aspects of the defining the proton's structure as well. We look forward to the future measurements which have the capability to go beyond broadly averaged quantities to true distributions of the gluon spin.

\section{REFERENCES}

1. Introduction to High Energy Physics, 4th Ed., D.H. Perkins, (Cambridge Univ. Press, Cambridge, 2000), p. 131.

2. B.W. Fillipone and X.D. Ji, Adv. Nucl. Phys., 26, 1 (2001) and references therein.

3. see S. Platchkov, this conference proceedings.

4. G. Bunce et al., Ann. Rev. Nucl. Part. Sci., 525 (2000).

5. M. Bai, The 17th Int. Spin Physics Symposium, Kyoto, Japan, Oct. 2006, in press.

6. K.H. Ackermann et al., Nucl. Instrum. Meth. A 499, 624 (2003).

7. J. Adams et al., the STAR Collab., Phys. Rev. Lett. 91 (2003) 072304.

8. G. Blazey et al., hep-ex/0005012.

9. B.I. Abelev, et al., the STAR Collab., hep-ex/0608030, submitted for publication.

10. B. Jäger et al., Phys. Rev. D70, 034010 (2004).

11. B. Surrow, The 17th Int. Spin Physics Symposium, Kyoto, Japan, Oct. 2006, in press. 